\title{
Efeitos da exposição à altitude sobre os aspectos neuropsicológicos: uma revisão da literatura
}

\section{Effects of exposure to altitude on neuropsychology aspects: a literature review}

\author{
Valdir de Aquino Lemos ${ }^{1,3}$, Hanna Karen Moreira Antunes ${ }^{2,3}$, Ronaldo Vagner Thomatieli dos Santos ${ }^{2,3}$, \\ Juliana Martuscelli da Silva Prado ${ }^{3}$, Sergio Tufik ${ }^{1,3,4}$, Marco Túlio De Mello ${ }^{1,3,4}$ \\ 1 Departamento de Psicobiologia, Universidade Federal de São Paulo (UNIFESP), São Paulo (SP), Brasil \\ 2 Departamento de Biociências, Campus da Baixada Santista, Universidade Federal de São Paulo (UNIFESP), Santos (SP), Brasil \\ 3 Centro de Estudos em Psicobiologia e Exercício (CEPE), São Paulo (SP), Brasil \\ 4 Conselho Nacional de Desenvolvimento Científico e Tecnológico (CNPq)
}

\section{Resumo}

Objetivo: Discutir os efeitos da exposição à altitude sobre as funções neuropsicológicas. Método: Foi realizada uma revisão de literatura usando como fonte de pesquisa artigos indexados no Pubmed, no período de 1921 a 2008, utilizando as palavras-chave "cognition and hypoxia", "hypoxia and neuropsychology", "acute hypoxia", "chronic hypoxia" e "acclimatization and hypoxia", além de livros específicos do assunto. Discussão: Os efeitos agudos e crônicos da hipóxia podem alterar inúmeras funções neuropsicológicas em diferentes altitudes, decorrentes de alteraçōes fisiológicas que resultam da diminuição parcial de oxigênio $\left(\mathrm{O}_{2}\right)$, que podem levar as alterações neuropsicológicas, como atenção, memória, tomada de decisão e demais funçôes executivas, em indivíduos expostos a grandes altitudes. Conclusão: Indivíduos que se expõem às grandes altitudes devem utilizar suplementação de $\mathrm{O}_{2}$ e prática de aclimatização, entre outras estratégias para minimizar os efeitos negativos da hipóxia nos aspectos neuropsicológicos.

Descritores: Hipóxia; Neuropsicologia; Altitude; Aclimatação; Prevenção de doenças

\begin{abstract}
Objective: Discuss the effects of altitude exposure on neuropsychological functions. Method: We have conducted a literature review using as source indexed articles at Pubmed in the period from 1921 to 2008, using the following key words: "cognition and hypoxia", "hypoxia and neuropsychology", "acute hypoxia", "chronic hypoxia", and "acclimatization and hypoxia", as well as specific books on the subject. Discussion: Acute and chronic effects of Hypoxia can alter many of the neuropsychological functions in different altitudes due to physiological changes resulted by the oxygen $\left(\mathrm{O}_{2}\right)$ partial decrease that can lead to neuropsychological alterations in individuals exposed to high altitudes. Conclusion: Individuals exposed to high altitudes must use an $\mathrm{O}_{2}$ supplementation and the practice of acclimatization, among other strategy ways that can be used in order to minimize the negative effects of hypoxia on neuropsychological aspects.
\end{abstract}

Descriptors: Hypoxia; Neuropsychology; Altitude; Acclimatization; Disease prevention

\section{Introdução}

Atualmente, há uma grande procura por práticas de atividades físicas relacionadas à altitude, como caminhada, montanhismo e esqui, no Brasil e em diversos outros países. Além disso, passeios e estadias em altitudes elevadas por períodos curtos ou prolongados a trabalho também são muito praticados ${ }^{1,2}$. A elevação da altitude faz com que a pressão barométrica em relação ao nível do mar diminua, resultando em uma redução da pressão parcial de oxigênio $\left(\mathrm{O}_{2}\right)$ para o organismo (sangue e tecidos corporais) ${ }^{3}$. A esta diminuição da parcialidade da oferta de $\mathrm{O}_{2}$ denomina-se hipóxia, responsável por respostas às grandes altitudes ${ }^{4}$. Portanto, de modo geral, os efeitos agudos e crônicos da hipóxia podem acarretar no ser humano alteraçôes fisiológicas e também cerebrais. ${ }^{5}$
Fisiologicamente, na vigência da exposição às grandes altitudes, o indivíduo tenta adaptar-se e o organismo humano produz respostas em vários sistemas, por isso acontecem diferentes ajustes que vão desde alteraçôes no sistema cardiovascular até o músculo esquelético, passando pelo endócrino, imune, até chegar ao cérebro $^{6}$. Tais alteraçôes fisiológicas, decorrentes da diminuição da oferta de $\mathrm{O}_{2}$, afetam a manutenção das funções cerebrais e físicas, que dependem de um percentual de $21 \%$ de $\mathrm{O}_{2}$ para funcionar adequadamente ${ }^{7}$.

Uma em cada oito pessoas que se propõem a escalar a maior montanha do mundo, por exemplo, o Monte Evereste, com $8.848 \mathrm{~m}$ de altitude, morre. De cada quatro pessoas que atingem 
o cume da montanha com sucesso, há pelo menos um que perde a vida durante a descida ou durante a subida logo após uma longa exposição à altitude. Todos os anos, uma parcela grande de pessoas também segue a outros lugares do mundo com altitudes elevadas, como Machu Picchu, Andes boliviano, Alpes suíços, entre outros ambientes que também podem colocar a vida de pessoas em risco em decorrência da hipóxia ${ }^{8}$.

Somente nas montanhas localizadas na região dos Himalaias, no período de 1950 a 2006, foi registrada uma média de 784 mortes em altitudes elevadas, o equivalente a 14 óbitos por ano?. Este dado é assustador, quando se compara com outro tipo de esporte considerado de alto risco, como, por exemplo, o boxe, que no período de 1890 a 2007, resultou em uma média de 923 óbitos, o equivalente a oito pessoas por ano em todo o mundo ${ }^{10}$. Tais dados indicam que os números de óbitos em ambientes de hipóxia podem ser até maiores do que aqueles gerados pela prática de esportes considerados de alto risco.

Assim, indivíduos expostos a 610-2.440m já podem apresentar alterações na aprendizagem ${ }^{11}$ e, em altitudes acima de $3.500 \mathrm{~m}$, podem ocorrer comportamentos ansiosos e sintomas da Doença Aguda da Montanha, tais como cefaléia, insônia, taquicardia, falta de ar e vertigens, semelhantes aos relatos de ataques de pânico ou de ansiedade severa. Esses comportamentos são ocasionados por uma hiperventilação em consequência da hipóxia, que pode levar a uma diminuição das concentrações de dióxido de carbono no sangue $\operatorname{arterial}^{12,13}$. Em altitudes de $5.000 \mathrm{~m}$, os efeitos da hipóxia podem produzir no organismo menor resistência muscular nos braços e nas pernas, dores de cabeça, tonturas, dificuldades para respirar e, além disso, alterações visuomotoras, mudanças de personalidade (como sintomas obsessivo-compulsivos e hostilidade, pelas diminuições da pressão de $\mathrm{O}_{2}$ inspirado pela traquéia), pressão alveolar de $\mathrm{O}_{2}$, pressão parcial de $\mathrm{O}_{2}$ na artéria sanguínea e pela saturação de hemoglobina com $\mathrm{O}_{2}$ no sangue arterial. $\mathrm{O}$ resultado da falta de $\mathrm{O}_{2}$ é, portanto, crucial como mecanismo no desenvolvimento de problemas físicos que podem levar às alteraçôes neuropsicológicas em grandes altitudes ${ }^{14-16}$. A 6.000m de altitude, a média de erros em avaliaçôes neuropsicológicas para indivíduos não aclimatizados são maiores em relação àqueles aclimatizados, porque a depressão vasomotora induzida pela hipóxia central pode proporcionar uma vasodilatação arterial e levar a alteraçôes cerebrais ${ }^{15}$. Já em uma altitude a $8.000 \mathrm{~m}$, pode ocorrer hipotermia ou morte súbita que estão associadas com edema cerebral vasogênico, predominantemente causado por uma falha na regulação endotelial do fluido vascular, logo após a diminuição extenuante de $\mathrm{O}_{2} \operatorname{arterial}^{16}$.

De fato, é evidente que o risco e o perigo são inerentes à exposição ambiental extrema e adversa ${ }^{17}$. Neste sentido, é indiscutível a importância deste assunto, ainda pouco estudado na literatura brasileira e relatado no âmbito da área da saúde. Assim, o objetivo da presente revisão é o de discutir os efeitos agudos e crônicos da exposição à altitude sobre as funções neuropsicológicas. Será dada ênfase às discussões dos efeitos da exposição à altitude sobre as funções neuropsicológicas, e diferenças entre determinadas populações envolvidas na resposta à altitude e os mecanismos de prevenção dos efeitos de exposição aguda e crônica à altitude.

\section{Método}

Para a realização deste estudo foi conduzida uma revisão integrativa da literatura, utilizando como fonte artigos indexados pela base de dados ISI e Medline e pesquisa em livros específicos que abordaram os aspectos relacionados à hipóxia e às alterações neuropsicológicas no período de 1921 a 2008.

As palavras-chave utilizadas para busca foram "cognition and hypoxia", "bypoxia and neuropsychology", "acute hypoxia", "chronic hypoxia" e "acclimatization and hypoxia", utilizando-se os boleanos específicos destas bases a fim de obter diversos arranjos de busca, maximizando tanto a abrangência quanto a qualidade da pesquisa.

Foram selecionados 61 artigos que descreveram os efeitos da altitude entre 610 e $8.848 \mathrm{~m}$, publicados nos últimos 88 anos, além de seis livros de modo a centrar os resultados deste trabalho nas contribuições mais atuais. Nenhum critério de exclusão foi utilizado.

Os estudos que investigaram os efeitos agudos e crônicos com tempo de exposição à altitude entre 30 minutos a dois anos foram realizados em câmaras hipobáricas ou em ambientes naturais de montanha. Em sua maioria, tratava-se de estudos com seres humanos e delineamentos transversais.

\section{Resultados e Discussão \\ 1. Efeitos da exposição aguda à hipóxia sobre aspectos neuropsicológicos}

A exposição em um curto período de tempo em ambientes de grandes altitudes caracteriza-se como exposição aguda à hipóxia, que pode se iniciar em minutos ou em poucas horas ${ }^{18,19}$. Um estudo que se propôs a avaliar as reações do sistema nervoso central aos efeitos da hipóxia com sete voluntários em um simulador de altitudes, equivalente a $3.048 \mathrm{~m}$ durante seis horas e meia, mostrou que, neste período de tempo, ocorreram prejuízos nas tarefas de performance, além de dores de cabeça, fraqueza e vertigen $s^{20}$.

Além de diversas alterações decorrentes da hipóxia, outro estudo conduzido por Qin et al. ${ }^{21}$ teve como objetivo avaliar o tempo de reação de 14 homens saudáveis, entre 18 e 20 anos de idade, expostos a $5.000 \mathrm{~m}$, revelando que os efeitos agudos da hipóxia podem alterar o processamento de informações de forma negativa. Diante disso, considerando a importância que o tempo de reação apresenta para aqueles que se expõem em ambientes com uma parcela diminuída de $\mathrm{O}_{2}$, estudos relatam que as alterações nos processos de informação podem levar o indivíduo a cometer erros em momentos inesperados, causando-lhe sérios problemas no momento de tomar uma decisão objetiva e precisa ${ }^{6,22}$.

Outras variáveis neuropsicológicas foram estudadas por Wu Xet al. ${ }^{23}$, que observaram os efeitos agudos da hipóxia na performance aritmética em 16 jovens do gênero masculino com idade de 16 anos, expostos a 300m (controle), e grupos experimentais (a $3.600 \mathrm{~m}, 4.400 \mathrm{~m}$ e $5.000 \mathrm{~m}$ ) em um simulador de altitudes. Como resultados deste estudo, os autores mostraram que a média de erros de calcular (adição e subtração) e o tempo de reação aumentaram significativamente depois da exposição a $3.600 \mathrm{~m}$ e $4.400 \mathrm{~m}$ por uma hora, e a $5.000 \mathrm{~m}$ por 30 minutos. Além desses problemas aritméticos e do tempo de reação, os efeitos agudos da hipóxia 
também podem alterar a memória de curto prazo considerando dois aspectos importantes: armazenamento de informações e velocidade de recuperação de informações ${ }^{24}$. Desta forma, a exposição aguda à altitude pode desencadear inúmeros problemas ao cérebro, dada sua maior sensibilidade aos efeitos da diminuição parcial de $\mathrm{O}_{2}$ do que aquela de outros órgãos do corpo ${ }^{4}$. Os efeitos agudos da hipóxia no organismo humano podem comprometer as funções executivas em indivíduos que até mesmo se expõem a altitudes moderadas de 2.000 a $4.500 \mathrm{~m}^{25,26}$.

Oito alpinistas experientes que ficaram expostos a uma altitude de $8.500 \mathrm{~m}$ sem $\mathrm{O}_{2}$ suplementar participaram voluntariamente de uma sessão de testes neuropsicológicos e, diante disso, foi possível observar um leve comprometimento cognitivo na memória e atenção ${ }^{27}$.

Por outro lado, Pavlicek et al. realizaram um estudo com o objetivo de avaliar as relaçōes entre funçôes cognitivas e variáveis cardiovasculares e respiratórias durante a exposição aos efeitos agudos e crônicos da hipóxia ${ }^{28}$. O estudo foi composto por sete voluntários de gênero masculino expostos a um total de duas horas aos efeitos da hipóxia, alocados em três grupos (G1 - 450-1.5003.000m; G2 - 450-3.000-4.500m; Controle - 450-650-650m). Foram despendidos 30 minutos para cada grupo no simulador de altitude, e 10 minutos para ajustar a pressão barométrica do simulador. Os resultados demonstraram não haver diferenças significativas entre fluência verbal, associação de palavras e performance de decisão, lobo frontal e funções emocionais, apesar do decréscimo da desaturação de $\mathrm{O}_{2}$ e da diminuição da pressão diastólica a $4.500 \mathrm{~m}$ correlacionada com a saturação de hemoglobina com $\mathrm{O}_{2}$ no sangue arterial, indicando o início da hipóxia central em termos de imparidade funcional do centro vasomotor. Os autores deste estudo concluíram que a preservação cognitiva pode ser explicada pelo pouco tempo de exposição à hipóxia que os voluntários foram submetidos.

Talvez um período de tempo maior ou uma exposição a uma altitude mais elevada trariam alterações nos mesmos aspectos cognitivos propostos pelo estudo citado anteriormente. No entanto, quando se fala em maior tempo despendido sob condições de hipóxia, os estudos realizados para observar os seus efeitos crônicos sobre os aspectos neuropsicológicos podem explicar melhor o que acontece com maior tempo de exposição.

\section{Efeitos da exposição crônica à hipóxia sobre aspectos neuropsicológicos}

A exposição prolongada por mais de um dia ou semanas em ambientes de hipóxia caracteriza-se como crônica ${ }^{29}$. Kennedy et al., em um dos seus experimentos, descreveram os efeitos crônicos da hipóxia em cinco semanas de exposição ${ }^{18}$. Diante este contexto, estudos mostraram que os efeitos crônicos da hipóxia sobre os aspectos neuropsicológicos podem estar associados a inúmeras alterações fisiológicas ${ }^{6}$, conforme se pode observar na Figura 1.

Um estudo com roedores mostrou que os efeitos crônicos de seis semanas de exposição à hipóxia, a $6.000 \mathrm{~m}$, diminuem a concentração das catecolaminas, (adrenalina, noradrenalina e dopamina) em diferentes estruturas cerebrais como estriatum, cerebelo, hipocampo e lobo frontal ${ }^{30}$. Além disso, este tipo de exposição à hipóxia também pode alterar as atividades do lobo parietal e do lobo pré-frontal, no sentido de não parear a integração multisensorial, causando distorções da percepção corporal, alucinações visuais, baixa inibição e baixa resistência ao estresse $^{31}$. Assim, pode-se observar que as alteraçóes ocorrem em diferentes regiōes do cérebro. No entanto, ainda há controvérsias sobre os prejuízos que podem estar associados aos efeitos de grandes altitudes nas funções neuropsicológicas ${ }^{32}$.

Diante dos estudos realizados até o presente momento, percebese que os efeitos da hipóxia podem ser diversos e o organismo humano pode sofrer inúmeras variações. Como exemplo disso, Shukitt et al. realizaram um estudo com indivíduos saudáveis que ficaram expostos a $4.300 \mathrm{~m}$ de altitude por mais de um dia (28 horas), revelando que os mesmos tiveram pensamento claro, mas também apresentaram vertigem, sonolência e tristeza ${ }^{33}$. Outro estudo avaliou as experiências perceptuais em um grupo de oito alpinistas expostos a uma altitude extrema a $8.500 \mathrm{~m}$, sem $\mathrm{O}_{2}$

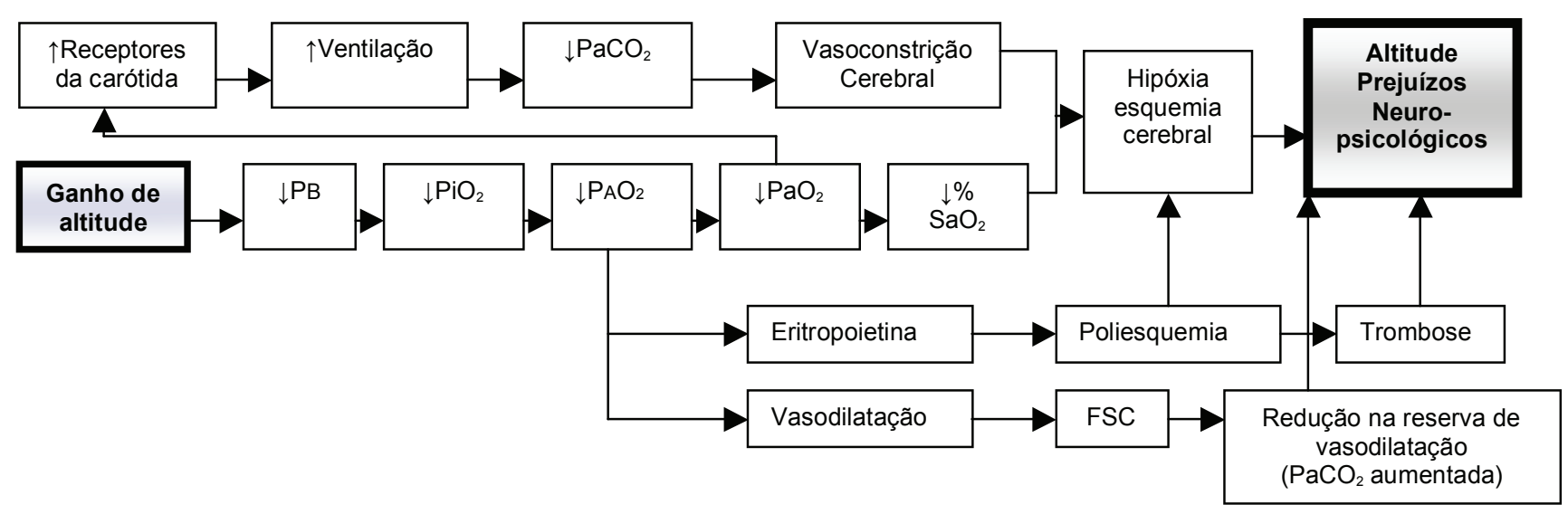

Figura 1 - Processos fisiológicos que levam ao entendimento dos prejuízos neuropsicológicos causados pela altitude

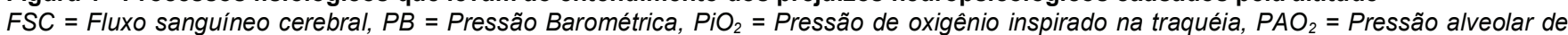
oxigênio, $\mathrm{PaO}_{2}=$ Pressão parcial de oxigênio na artéria sanguínea, $\mathrm{PaCO}_{2}=$ pressão parcial de dióxido de carbono na artéria sanguínea, \% $\mathrm{SaO}_{2}=$ Porcentagem da saturação de hemoglobina com oxigênio no sangue arterial. 
suplementar, durante uma semana, mostrando que os mesmos apresentaram ilusôes, distorçôes de esquema corporal, alucinaçôes visuais e auditivas, além de privação social e tensão ${ }^{34}$.

Além desses estudos relatados, a The American Medical Research Everest Expedition (1981) publicou dados nos quais encontraram prejuízos significativos dois anos após o término de uma expedição em grandes altitudes. Isso demonstra que a atenção voltada para este fenômeno deve ser maior ${ }^{35}$.

Seguindo a mesma linha de raciocínio, Regard et al. notaram que após 2-10 meses de volta ao nível do mar depois de uma exposição a $8.500 \mathrm{~m}$ de altitude sem oxigênio suplementar, por repetidas exposiçōes, as atividades neuropsicomotoras bem como concentração, memória de curto prazo e flexibilidade cognitiva foram alteradas significativamente em 25\% em alpinistas experientes com uma longa história de exposições a elevadas altitudes ${ }^{27}$.

Um estudo realizado em um simulador de altitudes a $8.848 \mathrm{~m}$ em que participaram como voluntários, oito alpinistas de gênero masculino entre 24 a 37 anos de idade, foi possível observar que as habilidades neuropsicomotoras, aprendizagem e a eficiência mental foram prejudicadas ${ }^{33,36}$. Assim, o mau funcionamento dos aspectos neuropsicomotores e outras alteraçôes nos processos de percepção, como tempo de reação e discriminação de cores, aprendizagem, atenção e memória, também são alterados nas condições de exposição à hipóxia crônica ${ }^{5,26,37}$.

Diante dos resultados descritos em contextos de altitudes elevadas citados até o presente momento, observa-se que são muitos os efeitos da hipóxia sobre o organismo humano (Tabela 1), como por exemplo, atrasos no tempo de reação sobre a execução de tarefas complexas, o que pode ser observado em estudos experimentais, incluindo expediçôes em altitudes elevadas na montanha ou em simulações com câmaras hipobáricas ${ }^{24,38-40}$. Prejuízos no tempo de reação também têm sido observados em baixas altitudes, como a $1.500 \mathrm{~m}^{41}$, embora as mais consistentes anormalidades sejam encontradas acima de $6.000 \mathrm{~m}^{26}$. Neste sentido, tanto em altas quanto em baixas altitudes os atrasos no tempo de reação podem refletir alterações importantes no processamento sensórioperceptual ${ }^{42-44}$. Portanto, este fenômeno pode resultar especificamente dos efeitos crônicos da hipóxia ou de outras variáveis atreladas ao percurso natural de grandes altitudes, como perturbações emocionais inerentes a estas condições, ausência de estímulos externos e exaustão física ${ }^{45}$.

\section{Variações étnicas dos efeitos da hipóxia sobre os aspectos neuropsicológicos}

Os efeitos da hipóxia sobre o corpo humano têm mostrado diferenças entre determinadas populações com relação à intensidade dos sinais e sintomas que diferem de uns em relação aos outros. Tais diferenças sugerem variaçôes fenotípicas nos sistemas de resposta à hipóxia e um grau de seleção natural na determinação dessas adaptações que estão associadas com uma maior sobrevivência ${ }^{53}$.

Tibetanos que vivem nos Himalaias quando expostos a condições extremas, parecem não exibir sinais e sintomas da hipóxia (apnéia obstrutiva do sono, aumento total no número de células vermelhas e dessaturação de hemoglobina) tão significativos nos indivíduos

Tabela 1 - Efeitos da hipóxia no cérebro

\begin{tabular}{|c|c|c|c|}
\hline Referências & Altitude (M) & Grupos de estudo & Resultados \\
\hline Malhota e Mathew, $1974^{46}$ & 4000 & 49 homens & Hiperatividade simpática e parassimpática \\
\hline Forster et al., $1975^{47}$ & 4300 & 7 homens & $\begin{array}{l}\text { Aumento na freqüência do EEG e redução do EEG } \\
\text { e respostas visuais evocadas }\end{array}$ \\
\hline Selvamurthy et al., $1978^{48}$ & 3500 & 30 soldados & Aumento da atividade alfa durante a aclimatização \\
\hline Townes et al., $1984^{49}$ & 8845 & 21 homens & $\begin{array}{l}\text { Alterações na habilidade de aprendizagem, } \\
\text { lembrança e expressão de informação verbal }\end{array}$ \\
\hline Vaernes et al., $1984^{20}$ & 3050 & 6 homens e 1 mulher & $\begin{array}{l}\text { Alterações na performance de testes } \\
\text { neuropsicológicos e de tarefas }\end{array}$ \\
\hline Fulco et al., $1989^{50}$ & 4300 & $\begin{array}{l}12 \text { jovens de gênero } \\
\text { masculino }\end{array}$ & $\begin{array}{l}\text { O bloqueio } \beta \text {-adrenérgico não exacerbou a AMS e } \\
\text { redução nos sentimentos de bem-estar }\end{array}$ \\
\hline Hornbien et al., $1989^{26}$ & $5400-8848$ & 40 homens & Declínio visual, verbal e na memória de longo prazo \\
\hline Kassirer e Von $1989^{51}$ & 7620 & 1 homem & Cefaléia e alterações na gustação \\
\hline Regard et al., $1989^{27}$ & $\geq 8500$ & 7 homens e 1 mulher & $\begin{array}{l}\text { Alterações na concentração, memória de curto } \\
\text { prazo e no controle de erros }\end{array}$ \\
\hline Cavaletti et al., $1990^{52}$ & 7000 & 9 homens e uma mulher & Alterações na performance da memória \\
\hline Wu Xet al., $1998^{23}$ & $3600-4400-5000$ & $\begin{array}{l}16 \text { jovens de gênero } \\
\text { masculino }\end{array}$ & $\begin{array}{l}\text { Erros de calcular (adição e subtração) e o tempo de } \\
\text { reação aumentaram }\end{array}$ \\
\hline Bolmont et al., $2000^{36}$ & 8848 & $\begin{array}{l}8 \text { alpinistas de gênero } \\
\text { masculino }\end{array}$ & $\begin{array}{l}\text { Habilidades neuropsicomotoras, aprendizagem e a } \\
\text { eficiência mental foram prejudicadas }\end{array}$ \\
\hline Qin et al., $2001^{21}$ & 5000 & $\begin{array}{l}14 \text { homens } \\
\text { saudáveis }\end{array}$ & Alterações no processamento de informações \\
\hline Pavlicek et al., $2005^{28}$ & $450-1500-3000-4500$ & 7 homens & $\begin{array}{l}\text { Não houve alterações entre fluência verbal, } \\
\text { associação de palavras e performance de decisão, } \\
\text { lobo frontal e funções emocionais }\end{array}$ \\
\hline
\end{tabular}

EEG - eletroencefalograma; $A M S$ - doença aguda da montanha 
que residem ao nível do mar, o que pode indicar uma melhor adaptação aos efeitos da hipóxia no organismo destas pessoas ${ }^{54,55}$.

O aumento total no número de células vermelhas é característico de comunidades andinas, enquanto nas comunidades tibetanas são observadas concentrações normais de hemoglobina ${ }^{56,57}$. Portanto, populações nativas que residem em locais de grandes altitudes são mais adaptadas às alterações ambientais proporcionadas pelos efeitos da hipóxia, como exemplificado pelo comportamento da saturação de $\mathrm{O}_{2}$ arterial, que em indivíduos originários das grandes altitudes é semelhante aos que nasceram e residem ao nível do $\operatorname{mar}^{53,54,58}$.

As adaptaçôes aos efeitos da hipóxia são mais rápidas nos tibetanos devido à herança autossômica dominante, ainda não conhecida, que está associada à aproximadamente $6 \%$ de maior saturação de $\mathrm{O}_{2}$.

Com a hipótese de que existem pessoas mais adaptáveis aos efeitos da hipóxia, Lieberman et al. realizaram um estudo no Monte Evereste em uma altitude de 5.300 a $8.000 \mathrm{~m}$, tendo como foco monitorar a fala e os déficits neuropsicológicos em um grupo de alpinistas $^{32}$. Alguns revelaram maior resistência à altitude do que outros alpinistas em relação à fala e aos déficits neuropsicológicos. Assim, percebe-se que, de acordo com a literatura, pode-se pensar em variações étnicas relacionadas à altitude. Por outro lado, novos estudos devem ser realizados para descrever com mais clareza e precisão a questão.

\section{Aclimatização e aclimatação}

O processo de aclimatização/aclimatação serve para o organismo humano se adaptar melhor às condições de hipóxia ${ }^{15,59}$. A aclimatização é conceituada como uma pré-adaptação ao meio inóspito que envolve diferentes aspectos naturais, como temperaturas negativas, solos não lineares, diminuição parcial de $\mathrm{O}_{2}$, entre outros fatores predominantes. Já o processo de aclimatação geralmente ocorre em locais que simulam altitude, por exemplo, câmaras hipobáricas, que proporcionam adaptaçôes fisiológicas para um único fator ambiental ou estressor (temperatura ou mudanças de altitude), que podem ser programadas pelo homem ${ }^{60}$.

A aclimatação pode ser importante para minimizar os efeitos da hipóxia em grandes altitudes, em função de uma pré-adaptação. Uma pesquisa realizada com o objetivo de avaliar a diminuição dos efeitos da hipóxia utilizando como estratégia a aclimatação em um simulador de altitudes, composto por quatro voluntários expostos a cinco dias em diferentes altitudes (nível do mar a 250m, 5.000m, $5.500 \mathrm{~m}, 6.000 \mathrm{~m}, 6.500 \mathrm{~m}$ e $7.000 \mathrm{~m}$ ), foi possível observar que períodos intermitentes de aclimataçóes sugerem uma eficiente pré-adaptação ao organismo humano ${ }^{61}$.

Em contrapartida, indivíduos não aclimatizados, depois de poucos minutos, podem apresentar alterações na visão, atenção, memória de curto prazo, habilidades aritiméticas e alterações na tomada de decisão. Conforme pode ser visto na Tabela 2, as capacidades neuropsicológicas em indivíduos não aclimatizados podem se alterar de acordo com mudanças de altitude - por exemplo, a $2.500 \mathrm{~m}$ acima do nível do mar, a sensibilidade visual está $83 \%$ de sua capacidade normal, atenção espacial $100 \%$, memória de curto prazo 97\%, habilidade aritmética 100\% e tomada de decisão também $100 \%$. Estes índices, comparados a $5.000 \mathrm{~m}$ acima do nível do mar, revelam uma mudança brusca em algumas das capacidades cognitivas: a sensibilidade visual corresponde a $48 \%$, atenção espacial a 57\%, memória de curto prazo a $76 \%$, habilidade aritmética a $86 \%$ e tomada de decisão a $90 \%{ }^{62}$. Esses resultados sugerem que quanto maior a exposição em grandes altitudes piores são as alterações neuropsicológicas proporcionadas pelos efeitos da hipóxia.

\section{Estratégias para prevenir os efeitos agudos e crônicos da hipóxia}

Em função da importância de prevenir ou minimizar os efeitos agudos e crônicos da hipóxia sobre os aspectos neuropsicológicos, algumas estratégias já descritas na literatura científica incluem a exposição gradual às grandes altitudes. A suplementação de $\mathrm{O}_{2}$ ou a aclimatização/aclimatação podem ser vistos como fatores relevantes na minimização dos efeitos deletérios da hipóxia ${ }^{63}$.

Suprimentos nutricionais como vitaminas C, E, L-carnitina e os oligoelementos (como zinco e antioxidantes), podem atuar como adjuvantes na minimização dos efeitos agudos e crônicos da hipóxia ${ }^{64-67}$. Portanto, deve ficar claro que tais estratégias não devem ser utilizadas única e exclusivamente como fator de proteção aos efeitos da hipóxia, lembrando que o organismo humano pode sofrer variações importantes diante deste contexto.

\section{Conclusão}

De modo geral, observa-se que os efeitos agudos e crônicos da hipóxia decorrentes da exposição a grandes e diferentes altitudes podem começar a alterar negativamente as inúmeras funções neuropsicológicas a partir de seis horas de exposição sucessiva. Neste sentido, sugere-se que os indivíduos que se expõem as grandes altitudes devem utilizar suplementação de $\mathrm{O}_{2}$

Tabela 2 - Alterações neuropsicológicos associadas com diferentes altitudes

\begin{tabular}{cccccc}
\hline Altitude $(\mathbf{m})$ & Sensibilidade visual & $\begin{array}{c}\text { Atenção } \\
\text { espacial }\end{array}$ & $\begin{array}{c}\text { Memória de curto } \\
\text { prazo }\end{array}$ & $\begin{array}{c}\text { Habilidades } \\
\text { aritméticas }\end{array}$ & Tomada de decisão \\
\hline 2500 & $83 \%$ & $100 \%$ & $97 \%$ & $100 \%$ & $100 \%$ \\
3500 & $67 \%$ & $83 \%$ & $91 \%$ & $95 \%$ & $98 \%$ \\
4200 & $56 \%$ & $70 \%$ & $83 \%$ & $92 \%$ & $95 \%$ \\
5000 & $48 \%$ & $57 \%$ & $76 \%$ & $86 \%$ & $90 \%$ \\
\hline
\end{tabular}


e prática de aclimatização, entre outras formas como estratégias para minimizar os efeitos negativos da hipóxia nos aspectos neuropsicológicos. Diante disso, espera-se que acidentes em ambientes de grandes altitudes sejam reduzidos. Ademais, com o número crescente de praticantes de montanhismo no Brasil, no qual a maior montanha apresenta cerca de $3.000 \mathrm{~m}$, os efeitos da exposição à altitude discutidos neste trabalho podem ser úteis não só para montanhistas, mas também para turistas e atletas que viajam para regiōes de elevadas altitudes, pois os efeitos negativos da hipóxia podem estar exacerbados caso não seja realizada uma boa adaptação do organismo nestas condiçôes.

\section{Agradecimentos}

Todos os autores são gratos a Associação Fundo de Incentivo à Psicofarmacologia (AFIP), Conselho Nacional de Desenvolvimento Científico e Tecnológico (CNPq), Fundo de Auxílio aos Docentes e Alunos (FADA), Centro de Estudos e Sonolência e Acidentes (CEMSA), Centros de Pesquisa, Expansão e Difusão do Instituto do Sono (CEPID/SONO), Fundação de Amparo à Pesquisa do Estado de São Paulo (FAPESP) - CEPID N98/143003-3 e Universidade Federal de São Paulo (UNIFESP) e, em especial, ao Centro de Estudos em Psicobiologia e Exercício (CEPE) e ao Centro de Estudos Multidisciplinar em Sonolência e Acidentes.

Financiamento e conflito de interesse

\begin{tabular}{|c|c|c|c|c|c|c|c|}
\hline $\begin{array}{l}\text { Membro do grupo } \\
\text { de autores }\end{array}$ & $\begin{array}{l}\text { Local de } \\
\text { trabalho }\end{array}$ & $\begin{array}{l}\text { Verba de } \\
\text { pesquisa }^{1}\end{array}$ & $\begin{array}{c}\text { Outro apoio à } \\
\text { pesquisa ou educação } \\
\text { médica continuada }\end{array}$ & $\begin{array}{c}\text { Honorários } \\
\text { de } \\
\text { palestrantes }\end{array}$ & $\begin{array}{l}\text { Participação } \\
\text { acionária }\end{array}$ & $\begin{array}{l}\text { Consultorl } \\
\text { Conselho } \\
\text { Consultivo }\end{array}$ & Outro $^{3}$ \\
\hline $\begin{array}{l}\text { Valdir de Aquino } \\
\text { Lemos }\end{array}$ & UNIFESP & - & - & - & - & - & - \\
\hline $\begin{array}{l}\text { Hanna Karen Moreira } \\
\text { Antunes }\end{array}$ & UNIFESP & - & - & - & - & - & - \\
\hline $\begin{array}{l}\text { Ronaldo Vagner } \\
\text { Thomatieli dos Santos }\end{array}$ & UNIFESP & - & - & - & - & - & - \\
\hline $\begin{array}{l}\text { Juliana Martuscelli da } \\
\text { Silva Prado }\end{array}$ & UNIFESP & - & - & - & - & - & - \\
\hline Sergio Tufik & UNIFESP & $\begin{array}{l}\text { FAPESP }^{* * *} \\
\text { CNPq }^{* * *}\end{array}$ & - & UNIFESP ${ }^{* * *}$ & - & - & - \\
\hline Marco Túlio de Mello & UNIFESP & - & - & - & - & - & - \\
\hline \multicolumn{8}{|c|}{$\begin{array}{l}\text { * Modesto } \\
\text { ** Significativa } \\
\text { *** Significativa. Montantes fornecidos à instituição do autor ou a colega onde o autor tem participação, não diretamente ao autor. } \\
\text { Nota: UNIFESP = Universidade Federal de São Paulo, FAPESP = Fundação de Amparo à Pesquisa do Estado de São Paulo; CNPq = } \\
\text { Conselho Nacional de Desenvolvimento Científico e Tecnológico. } \\
\text { Mais informações consultar as instruções aos autores }\end{array}$} \\
\hline
\end{tabular}

Referências

1. Magalhães J, Duarte J, Ascensão A, Oliveira J, Soares J. O desafio da altitude. Uma perspectiva fisiológica. Rev Port Cienc Desp. 2002;2(4):81-91.

2. Muhm JM, Rock PB, McMullin DL, Jones SP, Lu IL, Eilers KD, Space DR, McMullen A. Effect of aircraft-cabin altitude on passenger discomfort. N Engl J Med. 2007;357(1):18-27.

3. Rodway GW, Hoffman LA, MH, Sanders MH. High-altitude-related disorderspart I: pathophysiology, differential diagnosis, and treatment. Heart Lung. 2003;32(6):353-9.

4. Sharp RF, Bernaudin M. Hif1 and oxygen sensing in the brain. Nat Rev Neurosci. 2004;5(6):437-48.

5. Ortega VJ, Casal BG, Garrido E, Alcázar B. Neuropsychological functioning associated with high-altitude exposure. Neuropsychol Rev. 2004;14(4):197224.

6. Ortega VJ, Garrido E, Javierre C, Kloezeman CK. Human behaviour and development under high-altitude conditions. Dev Sci. 2006;9(4):400-10.

7. Sudarsky L. Pathophysiology of the nervous system. Boston: Little, Brown, and Company; 1990.

8. Loewenstein G. Because it is there: the challenge of mountaineering. Kyklos. 1999;52(3):315-44.

9. Salisbury R, Hawley E. The Himalaya by the numbers. A statistical analysis of mountaineering in the Nepal Himalaya 2007. [cited 28 dec 2008]. Available from: http:www.himalayandatabase.com

10. Svinth JR. Death under the spotlight: the Manuel Velazquez fatality collection a presentation. J Comb Sport. 2007;1(1):1-19.

11. Kelman GR, Crow TJ. Impairment of mental performance at a simulated altitude of 8000 feet. Aerosp Med. 1969;40(5):981-2.

12. Missoum G, Rosnet E, Richalet JP. Control of anxiety and acute mountain sickness in Himalayan mountaineers. Int J Sports Med. 1992;13(1):S37-9.
13. Walton T, Roth AG, Alicia EM, Georg WA, Eva M, Frank HW. High altitudes, anxiety, and panic attacks: is there a relationship? Depress Anxiety. 2002;16:(2)51-8.

14. Nelson M. Psychological testing at high altitudes. Aviat Space Environ Med.1982;53(2):122-6.

15. Koller EA, Bischoff M, Bührer A, Felder L, Schopen M. Respiratory, circulatory and neuropsychological responses to acute hypoxia in acclimatized and non-acclimatized subjects. Eur J Appl Physiol Occup Physiol. 1991;62(2):67-72.

16. Firth PG, Zheng H, Windsor JS, Sutherland AI, Imray $\mathrm{CH}$, Moore GW, Semple JL, Roach RC, Salisbury RA. Mortality on Mount Everest, 19212006: descriptive study. BMJ. 2008;337(111):a2654-60.

17. Ortner $S$. Thick resistance: death and the cultural construction of agency in Himalayan mountaineering. Representation. 1997;59(8):135-62.

18. Kennedy SL, Stanley WC, Panchal AR, Mazzeo RS. Alterations in enzymes involved in fat metabolismafter acute and chronic altitude exposure. $J$ Appl Physiol. 2001;90(1):17-22.

19. Curtis AS, Jerome A D. Control of breathing at high altitude. In: Hornbein FT, Schoene BR, editors. High altitude: an exploration of human adaptation. New York: Marcel Dekker; 2001. p.139-73.

20. Vaernes RJ, Owe JO, Myking O. Central nervous reactions to a 6.5 hours altitude exposure at 3.048 meters. Aviat Space Environ Med.1984;55(10):921-6.

21. Qin Y, Ma RS, Ni HY, Fu ZJ, Cheng HW. Locating the impairment of human cognitive function during hypoxia. Space Med Med Eng (Beijing). 2001;14(3):218-20.

22. Paintal SA. Cognitive functions in extraordinary environments. Indian J Med Res. 2004;120(2):73-4. 
23. Wu X, Li X, Han L, Wang T, Wei Y. Effects of acute moderate hypoxia on human performance of arithmetic. Space Med Med Eng (Beijing). 1998;11(6):391-5.

24. Fowler B, Prlic H. A comparison of visual and auditory reaction time and P300 latency thresholds to acute hypoxia. Aviat Space Environ Med. 1995;66(7):645-50.

25. Ernsting J. Prevention of hypoxia - acceptable compromises. Aviat Space Environ Med. 1978;49(3):495-502.

26. Hornbein TF, Townes BD, Schoene RB, Sutton JR, Houston CS. The cost the central nervous system of climbing to extremely higt altitude. $N$ Engl J Med. 1989;321(25):1714-9.

27. Regard M, Oelz O, Brugger P, Landis T. Persistent cognitive impairment in climbers after repeated exposure to extreme altitude. Neurology. 1989;39(2 Pt 1):210-3

28. Pavlicek V, Schirlo C, Nebel A, Regard M, Koller EA, Brugger P. Cognitive and emotional processing at high altitude. Aviat Space Environ Med. 2005;76(1):28-33.

29. Singh SB, Thakur L, Anand JP, Yadav D, Amitab, Banerjee PK. Effect of chonic hypobaric hypoxia on components of human event related potential. Indian J Med Res. 2004;120(2):94-7.

30. Ohkuwa T, Itoh H, Yamamoto T, Minami C, Yamazaki Y, Yoshida R, Kimoto, $\mathrm{S}$. Effects of long-term hypoxia and hypoxic exercise on brain monoamine levels in rat. Auton Neurosci. 2003;106(2):98-102.

31. Arzy S, Idel M, Landis T, Blanke O. Why revelations have occurred on mountains? Linking mystical experiences and cognitive neuroscience. Med Hypotheses. 2005;65(5):841-5.

32. Lieberman P, Morey A, Hochstadt J, Larson M, Mather S. Mount Everest A: space analogue for speech monitoring of cognitive deficits and stress. Aviat Space Environ Med. 2005;76(6 Suppl):B198-207.

33. Shukitt BL, Banderet LE. Mood states at 1600 and 4300 meters terrestria altitude. Aviat Space Environ Med. 1988;59(6):530-2.

34. Brugger P, Regard M, Landis T, Oelz O. Hallucinatory experiences in extreme-altitude climbers. Neuropsychiatry Neuropsychol Behav Neurol. 1999; 12(3):67-71

35. West JB. Do climbs to extreme altitude cause brain damage? Lancet. 1986;2(8503):387-8.

36. Bolmont B, Thullier F, Abraini JH. Relationships between mood and performances in reaction time, psychomotor ability, and mental afficiency during a 31-day gradual decompression in a hypobaric chamber from sea level to $8848 \mathrm{~m}$ equivalent altitude. Physiol Behav. 2000;71(5): 469-6.

37. Gilbson GE, Pulsinelli W, Blass JP, Duffy TE. Brain dysfunction in mild to moderate hypoxia. Am J Med. 1981;70(6):1247-4.

38. Bolmont B, Bouquet C, Thullier F. Relationship of personality traits with performance in RT, psychomotor ability, and mental eficiency during a 31-day simulated climb of Mount Everest in a hypobaric chamber. Percept Mot Skills. 2001;92(3 Pt 2):1022-30.

39. Kramer AF, Colcombe SJ, McAuley E, Scalf PE, Erickson KI. Fitness ageing and neurocognitive function. Neurobiol Aging. 1999;26(1):124-7.

40. Mackintosh JH, Thomas DJ, Olive JE, Chesner IM, Knight RJ. The effect of altitude on tests of reaction time and alertness. Aviat Space Environ Med. 1988;59(3):246-8.

41. Denison DM, Ledwith F, Poulton EC. Complex reaction times at simulated cabin altitudes at 5000 feet and 8000 feet. Aerosp Med. 1966;37(10):1010-3.

42. Hayashi R, Matsuzawa Y, Kubo K, Kobayashi T. Effects of simulated high altitude on event-related potential (P300) and auditory brain-stem responses. Clin Neurophysiol. 2005;116(6):1471-6.

43. Kida M. Psychophysiological studies under simulated high altitude. Japan J Psychonomic Sci. 1997;16(5):37-44.

44. Soltani M, Knight RT. Neural origins of the P300. Crit Rev Neurobiol. 2000;14(3-4):199-224.

45. Garrido E, Javierre C, Ventura JL, Segura R. Hallucinatory experiences at high altitude. Neuropsychiatry Neuropsychol Behav Neurol. 2000;13(2): 148 .

46. Malhotra MS, Mathew L. Effect of prolonged stay at altitude $(4000 \mathrm{~m})$ on autonomic balance. Aerosp Med. 1974;45(8):869-72.

47. Forster HV, Soto RJ, Dempsey JA, Hosko MJ. Effect of sojourn at 4,300 m altitude on electroencephalogram and visual evoked response. $J$ Appl Physiol. 1975;39(1):109-13.

48. Selvamurthy W, Saxena RK, Krishnamurthy N, Suri ML, Malhotra MS. Changes in EEG pattern during acclimatization to high altitude (3500 m) in man. Aviat Space Environ Med. 1978;49(8):968-71.
49. Townes BD, Horbien TF, Schoene RB, Sarnquist FH, Grant I. Human cerebral functions at extreme altitude. In: West L, Lahiri G, editors. High altitude and man. Bethesda: American Physiological Society; 1984. p.123-7.

50. Fulco CS, Rock PB, Reeves JT, Trad LA, Young PM, Cymerman A. Effects of propranolol on acute mountain sickness (AMS) and well-being at 4,300 meters of altitude. Aviat Space Environ Med. 1989;60(7):679-83.

51. Kassirer MR, Such RV. Persistent high-altitude headache and aguesia without anosmia. Arch Neurol. 1989;46(3):340-1.

52. Cavaletti G, Garavaglia P, Arrigoni G, Tredici G. Persistent memory impairment after high altitude climbing. Int J Sports Med. 1990;11(3):176-8.

53. Beall CM, Song K, Elston RC, Goldstein MC. Higher offspring survival among Tibetan women with high oxygen saturation genotypes residing at 4,000 m. Proc Natl Acad Sci USA. 2004;101(34):14300-4.

54. Beall CM, Strohl KP, Blangero J, Williams-Blangero S, Decker MJ, B0rittenham GM, Goldstein MC. Quantitative genetic analysis of arterial oxygen saturation in Tibetan highlanders. Hum Biol. 1997;69(5):597-604.

55. Niermeyer S, Yang P, Shanmina M, Drolkar R, Zhuang J, Moore LG. Arterial oxygen saturationin Tibetan and Han infants born in Lhasa, Tibet. $N$ Engl J Med. 1995;333(19):1248-52.

56. Beall CM. Tibetan and Andean patterns of adaptation to high-altitude hypoxia. Hum Biol. 2000;72(1):201-28.

57. Niermeyer S, Zamudio S, Moore LG. The people. In: Hornbein TF, Schoene RB, editors. High altitude: an exploration of human adaptation. New York: Marcel Dekker; 2001. p.43-100.

58. Beall CM, Blangero J, Williams-Blangero S, Goldstein MC. Major gene for percent of oxygen saturation of arterial hemoglobin in Tibetan highlanders. Am J Phys Anthropol. 1994;95(3):271-6.

59. Koller EA, Lesniewska B, Bührer A, Bub A, Kohl J. The effects of acute altitude exposure in Swiss highlanders and lowlanders. Eur J Appl Physiol Occup Physiol. 1993;66(2):146-54.

60. Piantadosi AC. The biology of human survival: life and death in extreme environments. New York: Oxford University Press; 2003.

61. Leiffen D, Poquin D, Savourey G, Barraud PA, Raphel C, Bittel J. Cognitive performance during short acclimation to severe hypoxia. Aviat Space Environ Med. 1997;68(11):993-7.

62. McFarland RA. Psychophysiological implications of life at altitude and including the role of oxygen in the process of aging. In: Yousef MK, Horvath SM, editors. Physiological adaptations: desert and mountain. New York: Academic Press; 1972. p.157-81.

63. Liu C, Smith TG, Balanos GM, Brooks J, Crosby A, Herigstad M, Dorrington KL. Robbins PA. Lack of involvement of the autonomic nervous system in early ventilatory and pulmonary vascular acclimatization to hypoxia in humans. $J$ Physiol. 2007;579(1):215-25.

64. Fenella JK, Avijit KD. Hypoxic adaptation during development: relation to pattern of neurological presentation and cognitive disability. Dev Sci. 2006;9(4):411-27.

65. Devi SA, Vani R, Subramanyam MV, Reddy SS, Jeevaratnam K. Intermittent hypobaric hypoxia-induced oxidative stress in rat erythrocytes: protective effects of vitamin E, vitamin C, and carnitine. Cell Biochem Funct. 2007;25(2):221-31.

66. Schreiber M, Trojan S. Protective effects of vitamins $\mathrm{C}$ and $\mathrm{E}$ in hypoxia. $S b$ Lek. 1995;96(2):163-6.

67. Dutta A, Ray K, Singh, VK, Vats, P, Singh, SN, Singh, SB. L-carnitine supplementation attenuates intermittent hypoxia-induced oxidative stress and delays muscle fatigue in rats. Exp Physiol. 2008;93(10):1139-46. 